\title{
Special Issue of Digestion: Inflammatory Bowel Disease
}

In the past 20 years, we have been witnessing astonishing achievements in the field of inflammatory bowel diseases (IBDs) both in the understanding of pathophysiology and in the development of new treatment options and clinical knowledge. This fast development and the overwhelming number of publications in the field make it necessary to provide the medical community with up-to-date summaries and reviews of the current literature.

Eight years ago we published a widely appreciated supplementary on the management of IBD in Digestion. Since then a large number of landmark studies have been published on new therapeutic avenues, disease monitoring, and complication management. New classes of drugs such as anti-integrin antibodies, anti-IL23 antibodies, Janus Kinase inhibitors, and mesenchymal stem cells have been introduced into the IBD doctor's armamentarium.

We now aim for earlier diagnosis, better patient stratification, targeted therapies, and individualized care. Besides that, we pay better attention to extraintestinal man- ifestations and unspecific symptoms such as pain and fatigue. We discuss mucosal healing and even histological healing and whether those goals can be achieved.

This, however, means that the strategies and algorithms for the management of IBDs become more complex, require more knowledge, and demand continuous education and training. For these purposes, we felt that it is time for another special issue of Digestion following the well-perceived last one in 2012.

We certainly hope that this new special issue fulfills your expectations and represents a useful update on the clinical management of our patients with IBD.

\section{Disclosure Statement}

The authors have no conflicts of interest to declare.

Gerhard Rogler, Zurich Alexander König, Göttingen Stephan R. Vavricka, Zurich 